\title{
Isolation and characterisation of phages targeting clinical Pseudomonas aeruginosa carrying virulence genes
}

\author{
Nur Afifah Binti Jamil¹, Abeer Ameen Baqer¹, Siti Noor Adnalizawati Adnan², Ainulkhir Hussin ${ }^{3}$, Norefrina \\ Shafinaz Md. Nor ${ }^{*}$
}
${ }^{1}$ School of Biosciences and Biotechnology, Faculty of Science and Technology, Universiti Kebangsaan Malaysia, 43600 Bangi, Selangor, Malaysia.
${ }^{2}$ Faculty of Dentistry, Universiti Sains Islam Malaysia, Bandar Baru Nilai, 71800 Nilai, Negeri Sembilan, Malaysia. ${ }^{3}$ Department of Pathology, Queen Elizabeth Hospital, 88586 Kota Kinabalu, Sabah, Malaysia.
Email: efrina@ukm.edu.my

\begin{abstract}
Aims: The aim of the study was to isolate and characterise bacteriophages specific to Pseudomonas aeruginosa carrying virulence genes.

Methodology and results: Four clinical strains of $P$. aeruginosa CL1, CL2, CL3 and CL4 were obtained from Queen Elizabeth Hospital, Kota Kinabalu, Sabah. The bacterial strains were screened for virulence genes exoS, toxA and oprl and biofilm production. Six P. aeruginosa specific bacteriophages, namely PAtk1, PAtk2, PAtk3, PAtk4, PAtk5 and PAtk6, were isolated from Tasik Kejuruteraan, Universiti Kebangsaan Malaysia. These bacteriophages were screened for lytic spectrum against $P$. aeruginosa and two species of Enterobacteriaceae (Escherechia coli and Salmonella Typhi). PCR results showed that all strains possessed exoS, toxA and oprl genes except CL2 that lacked exoS. Nevertheless, it was CL2 that produced the highest biofilm density. Further, based on Transmission Electron Microscopy, PAtk15 and PAtk6 were classified into the family Myoviridae and Siphoviridae, respectively. Among all six isolated phages, only PAtk4 and PAtk6 showed the broadest lytic spectrum in which lytic activity was observed against all clinical $P$. aeruginosa strains.

Conclusion, significance and impact of study: In this study we reported the isolation of six bacteriophages from Myoviridae and Siphoviridae that are specific to $P$. aeruginosa possessing exoS, tox $A$ and oprl genes. Bacteriophages Patk4 and PAtk6 were able to infect all four strains of $P$. aeruginosa, making these phages potential agents in combating infections by the bacterium.
\end{abstract}

Keywords: Myoviridae, Siphoviridae, exoS, toxA, oprl

\section{INTRODUCTION}

Pseudomonas aeruginosa causes various nosocomial infections, including urinary tract infections, pneumonia, infections of burns and bacteremia (Holguín et al., 2015). Infections by this pathogen are frequent in patients with immune deficiency or diabetes. It is the primary pathogen responsible for a high percentage of death due to sepsis (Young, 1984). The bacterium is the most common hospital-acquired infecting agent and leading cause of respiratory infection in cystic fibrosis patients (Bendiak and Ratjen, 2009). Infections tend to become chronic when the infecting strain converts to colonisation mode, which includes production of biofilms that are hindered by attacks from antibodies, host phagocytes and some antibiotics, promoting development of resistant variants (Breidenstein et al., 2011). A recent study mentioned the close relationship between biofilm formation and the bacterium drug resistance pattern in which Extended
Spectrum Beta-Lactamases (ESBL)-producing $P$. aeruginosa that form biofilms tend to be more pathogenic compared to its non-ESBL producing counterpart (Ullah et al., 2017). The resistant pathogen may gain access to the bloodstream and subsequently cause death due to the production of endo- and exotoxins (Liu, 1974). In addition to their intrinsic resilience towards antibiotic treatment, $P$. aeruginosa are increasingly found to contain multidrugresistant plasmids (Nordmann et al., 2007). There is also a variety of virulence factors produced by $P$. aeruginosa, including enzymes that promote tissue invasion and extracellular polymers that form biofilms (Pollack, 1984).

In this study, we focused on the most significant virulence factors encoded by exoS, toxA and oprl in $P$. aeruginosa. exoS gene encodes for an ADPribosyltransferase, namely exoenzyme $S$, which is secreted directly through a type-III secretion system into the cytosol of host epithelial cells (Galle et al., 2012). Furthermore, this gene was found to be frequently 
Malays. J. Microbiol. Vol 14(6) Special Issue 2018, pp. 563-569 DOI: http://dx.doi.org/10.21161/mjm.1461815

present in drug resistant isolates (Fazeli and Momtaz, 2014). toxA, considered as one of the major extracellular virulence factors produced by $P$. aeruginosa, encodes for exotoxin A protein which is also an ADP-ribosyl transferase. Expression of this gene permanently inhibits protein synthesis in eukaryotic cells, which results in cell mortality (Iglewski et al., 1975). Further, oprl encodes for one of the major outer membrane proteins in $P$. aeruginosa that is crucial for bacterial interactions with the surrounding environment (Khan and Cerniglia, 1994).

It is suggested that antibiotic resistant bacteria will kill 10 million people per year by 2050 , more than the number of mortalities caused by cancer (O'Neill, 2014). Thus, this serves as a signal to develop new classes of antimicrobial therapies. Bacteriophages have been explored as a biological alternative or supplement to the current antibiotic therapy for Pseudomonas infections (Viertel et al., 2014). Here, we describe the detection of virulence genes exoS, toxA and oprl in $P$. aeruginosa and the isolation and characterisation of freshwater lake-derived bacteriophages specific against the bacterium.

\section{MATERIALS AND METHODS}

\section{Bacterial strains}

Clinical isolates of $P$. aeruginosa CL1, CL2, CL3 and CL4 were obtained from the Pathology Laboratory, Queen Elizabeth Hospital, Kota Kinabalu, Sabah, Malaysia. In addition, Escherichia coli UKMCC 1006 and Salmonella Typhi UKMCC 1013 (obtained from Universiti Kebangsaan Malaysia Culture Collection) were used to observe the infection spectrum by the isolated bacteriophages.

\section{Bacterial genomic DNA extraction}

DNA from $P$. aeruginosa CL1, CL2, CL3 and CL4 was extracted through boiling method (Holmes and Quigley, 1981). One or two bacterial colonies grown on an agar plate was collected and suspended into a $1.5 \mathrm{~mL}$ microcentrifuge tube containing sterile $0.25 \mathrm{~mL}$ DNase/RNase-free water. The bacterial suspension was then boiled for 10 to $15 \mathrm{~min}$ and centrifuged at $10,000 \times g$ at $4{ }^{\circ} \mathrm{C}$ for $10 \mathrm{~min}$. The obtained supernatant containing DNA was collected and stored at $-20{ }^{\circ} \mathrm{C}$ prior to PCR amplification.

\section{Detection of virulence genes in $P$. aeruginosa CL1, CL2, CL3 and CL4 by PCR}

Polymerase chain reaction (PCR) was performed by using specific primers to detect exoS, toxA and oprl virulence genes (Al-Kaaby, 2015) (Table 1). Following optimisation, the PCR mixture was set up as follows: GoTaq ${ }^{\circledR}$ Reaction Buffer $1(10 \mu \mathrm{L})$, PCR Nucleotide Mix, $10 \mathrm{mM}$ each $(1.0 \mu \mathrm{L})$, upstream primer $(1.0 \mu \mathrm{L})$, downstream primer $(1.0 \mu \mathrm{L})$, GoTaq ${ }^{\circledR}$ DNA Polymerase $(0.25 \mu \mathrm{L})$, template DNA $(5 \mu \mathrm{L}$ of $<0.5 \mu \mathrm{g} / 50 \mu \mathrm{L})$ and nuclease-free water (Promega Corp., USA) for a $50 \mu \mathrm{L}$ final volume. The reaction mixtures were subject to the following empirically optimised thermal cycling parameters: initial denaturation temperature of $95^{\circ} \mathrm{C}(5$ min); followed by 30 cycles of denaturation at $95^{\circ} \mathrm{C}(30$ $\mathrm{sec})$, annealing $58^{\circ} \mathrm{C}(30 \mathrm{~s})$ and extension $72{ }^{\circ} \mathrm{C}(1 \mathrm{~min})$ and then final extension at $72{ }^{\circ} \mathrm{C}$ (10 min). Negative control (water) was included in every set of PCR reaction.

Table 1: List of primer sequences used in PCR to detect virulence genes exoS, toxA and oprl.

\begin{tabular}{|c|c|c|c|}
\hline Primer & $\begin{array}{l}\text { Size } \\
\text { (bp) }\end{array}$ & Sequence & $\begin{array}{l}\text { Gene Bank } \\
\text { code }\end{array}$ \\
\hline exos & 684 & $\begin{array}{l}\text { F: } \\
\text { GCTTCAGCAGAGTCC } \\
\text { GTCTT } \\
\text { R: } \\
\text { GCCGATACTCTGCTG } \\
\text { ACCTC }\end{array}$ & L27629.1 \\
\hline toxA & 487 & $\begin{array}{l}\text { F: } \\
\text { GGCTATGTGTTCGTC } \\
\text { GGCTA } \\
\text { R: } \\
\text { TGATCGCCTGTTCCTT } \\
\text { GTCG }\end{array}$ & AF227424.1 \\
\hline oprl & 202 & $\begin{array}{l}\text { F: } \\
\text { CGGCTGGGAGATTGC } \\
\text { TGTTA } \\
\text { R: } \\
\text { CCTTGCGATAGGCTT } \\
\text { CGTCA }\end{array}$ & X58714.1 \\
\hline
\end{tabular}

\section{Detection of amplicons}

Following amplification, aliquots $(5 \mu \mathrm{L})$ were removed from each reaction mixture and examined by gel electrophoresis $(80 \mathrm{~V}, 45 \mathrm{~min}$ ) in gels composed of $1 \%$ $(\mathrm{v} / \mathrm{w})$ agarose containing gel red dye in TAE $(1 \times)$ buffer. Gels were visualised under UV illumination and all images obtained were archived. The result was considered positive for Pseudomonas if the three genes resulted in an amplicon of the expected size via electrophoresis.

\section{P. aeruginosa biofilm biomass quantification}

Biofilm biomass quantification of $P$. aeruginosa strains was conducted using colorimetric micro-titer plate assay. Each $P$. aeruginosa strain was grown overnight at $37^{\circ} \mathrm{C}$ in Luria Bertani (LB) broth. The overnight culture $(50 \mu \mathrm{L})$ was inoculated into $5 \mathrm{~mL}$ of LB broth and grown at $37^{\circ} \mathrm{C}$ until it reached an approximate Optical Density $\left(\mathrm{OD}_{600}\right)$ of $0.5-0.7$. The culture $(10 \mu \mathrm{L})$ was then transferred into a well (of a 96-well plate) containing $190 \mu \mathrm{L}$ LB broth and incubated at $37{ }^{\circ} \mathrm{C}$ overnight. Cells were washed with phosphate saline buffer (PBS) twice and air dried before adding crystal violet $(200 \mu \mathrm{L}, 1 \%(\mathrm{w} / \mathrm{v})$ and left at room temperature $\left(25^{\circ} \mathrm{C}\right)$ for $15 \mathrm{~min}$. Excess crystal violet was removed and the well plate was washed twice with PBS and air dried. The bound crystal violet stain was dissolved by using $33 \%(\mathrm{v} / \mathrm{v})$ glacial acetic acid and left to stand for 5 min before transfer to clean well. Absorbance of the 
Malays. J. Microbiol. Vol 14(6) Special Issue 2018, pp. 563-569 DOI: http://dx.doi.org/10.21161/mjm.1461815

biofilm biomass was measured at $570 \mathrm{~nm}$ using ELISA reader. All experiments were conducted in triplicates with sterile LB broth as negative control.

\section{Phage isolation}

Bacteriophages were isolated using the double agar overlay method with $0.75 \%(\mathrm{w} / \mathrm{v})$ LB agar where $P$. aeruginosa UKMCC 1011 was used as the indicator strain. Water samples (obtained from Tasik Kejuruteraan, Universiti Kebangsaan Malaysia, Bangi, Malaysia) was centrifuged at $6000 \mathrm{rpm}$ for $10 \mathrm{~min}$ before filtering the supernatant through a $0.22 \mu \mathrm{m}$ sterile syringe filter. For phage propagation, a mixture of $100 \mu \mathrm{L}$ of water sample and $200 \mu \mathrm{L}$ of overnight bacterial culture were added to 5 $\mathrm{mL}$ of $0.75 \%(\mathrm{w} / \mathrm{v})$ top LB agar and poured onto $1.5 \%$ $(\mathrm{w} / \mathrm{v})$ bottom LB agar and incubated at $37^{\circ} \mathrm{C}$ overnight. Formation of plaques was examined the following day. For negative control, Salt-Magnesium (SM) buffer was used as a mock bacteriophage and added to the molten agar. The harvested bacteriophages were selected according to their plaque morphology. Several rounds of plaque purification were performed to obtain pure bacteriophage suspensions. Purified plaques were then tested against $P$. aeruginosa CL1, CL2, CL3 and Cl4.

\section{Preparation of concentrated phage stock}

Purified bacteriophages were serially diluted in SM buffer and plated with host bacteria before overnight incubation at $37^{\circ} \mathrm{C}$. Plates with the highest number of plaques were chosen. After adding SM buffer $(5 \mathrm{~mL})$, each plate was left at room temperature $\left(25^{\circ} \mathrm{C}\right)$ and swirled every 10 minutes for at least $1 \mathrm{~h}$. The liquid and top agar layer were transferred into a sterile tube, vortexed and centrifuged at $1300 \times g(10 \mathrm{~min})$ to remove agar residues. The obtained supernatant was filtered through $0.22 \mu \mathrm{m}$ sterile membrane filter and stored in $50 \%(\mathrm{v} / \mathrm{v})$ glycerol at $-80{ }^{\circ} \mathrm{C}$ and in SM buffer at $4{ }^{\circ} \mathrm{C}$. Stock titres were determined by double-layer agar method (Swanstrom and Adams, 1951).

\section{Host spectrum determination for bacteriophages}

$P$. aeruginosa CL1, CL2, CL3, CL4, E. coli UKMCC 1006 and S. Typhi UKMCC 1013 cultures were each (24-hour grown, $200 \mu \mathrm{L}$ ) inoculated into $5 \mathrm{~mL}$ of $0.75 \%(\mathrm{w} / \mathrm{v})$ top LB agar and overlaid on $1.5 \%$ bottom LB agar. Each overlay was allowed to solidify for $15 \mathrm{~min}$ before $5 \mu \mathrm{L}$ of phages were dropped onto the solidified $0.75 \%$ top LB agar. The agar was dried prior to incubation at $37{ }^{\circ} \mathrm{C}$ overnight. Negative control consists of each bacterial strain in sterile SM buffer without bacteriophages. A positive result was indicated by the formation of clear zones on bacterial lawns.

\section{Transmission Electron Microscopy (TEM)}

Bacteriophage particles were negatively stained and examined by Electron Microscopy at Electron Microcsopy Laboratory of Universiti Kebangsaan Malaysia. Staining was performed by firstly depositing phage suspension on copper grids with carbon-coated Formvar film and subsequent staining with $2 \%(\mathrm{v} / \mathrm{v})$ uranyl acetate $(\mathrm{pH} 4.0)$. Phage morphologies were examined using Transmission Electron Microscope (Philips CM12 TEM). Each phage was characterised based on their morphology according to Ackermann (2011)

\section{RESULTS}

Detection of virulence genes exoS, toxA and oprl in and biofilm biomass quantification of $P$. aeruginosa CL1, CL2, CL3 and CL4

The presence or absence of $P$. aeruginosa virulence genes exoS, toxA and oprl in $P$. aeruginosa CL1, CL2, CL3 and CL4 strains are shown through electrophoresis gel images in Figures 1-3, respectively. Based on the images, all virulence genes were detected in all four strains except for exoS that was not observed in CL2. This shows that CL2 was unable to produce exoenzyme $S$ encoded by the exoS gene.

Overnight biofilm formation by the $P$. aeruginosa strains was quantified through crystal violet assay. Amongst all strains, CL2 had the highest amount of absorbance $\left(\mathrm{OD}_{570}\right)(1.93 \pm 0.27)$ followed by CL1, CL3 and CL4 (1.06 $\pm 0.32,0.35 \pm 0.05,0.25 \pm 0.14$, respectively) (Table 2 )

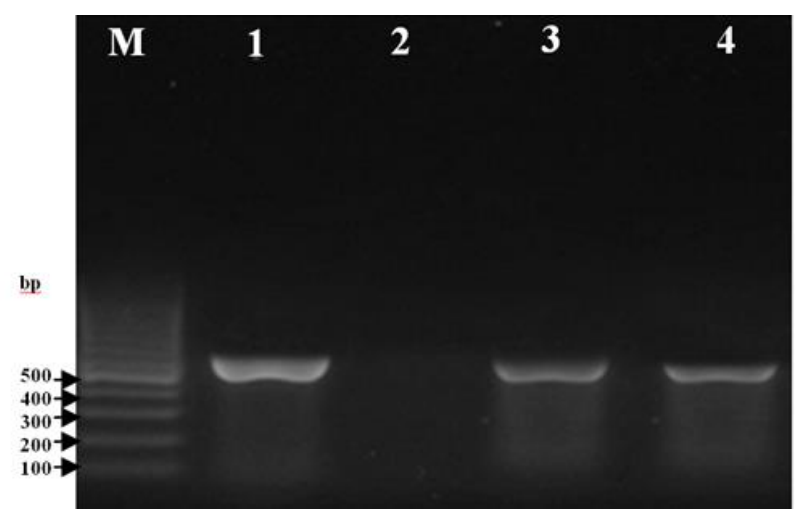

Figure 1: PCR amplification of exoS gene (684 bp) separated by electrophoresis on a $1 \%$ agarose gel, stained with red dye at 80 volts/cm for $1 \mathrm{~h}$. Photographed under UV light. 1000 bp DNA marker (M); (1) $P$. aeruginosa CL1; (2) $P$. aeruginosa CL2; (3) $P$. aeruginosa CL3; (4) $P$. aeruginosa CL4. 
Malays. J. Microbiol. Vol 14(6) Special Issue 2018, pp. 563-569 DOI: http://dx.doi.org/10.21161/mjm.1461815

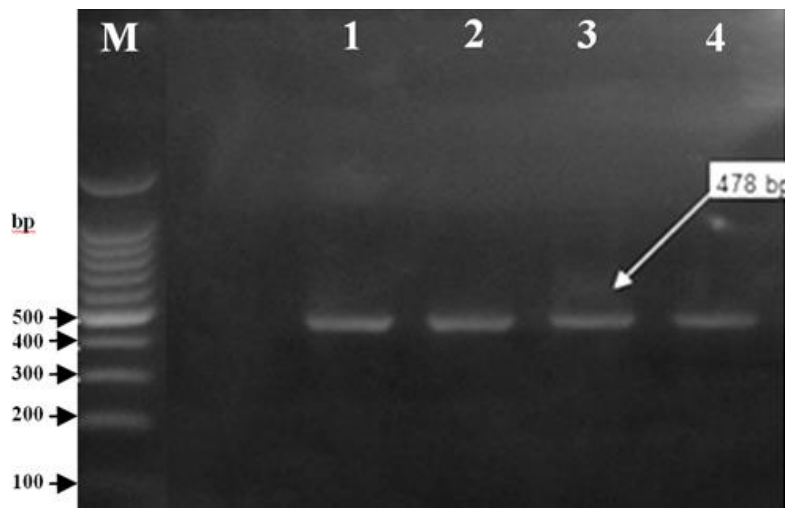

Figure 2: PCR amplification of toxA gene (478 bp) separated by electrophoresis on a $1 \%$ agarose gel, stained with red dye at 80 volts $/ \mathrm{cm}$ for 1 hour. Photographed under UV light. 1000 bp DNA marker (M); (1) $P$. aeruginosa CL1; (2) $P$. aeruginosa CL2; (3) $P$. aeruginosa CL3; (4) $P$. aeruginosa CL4.

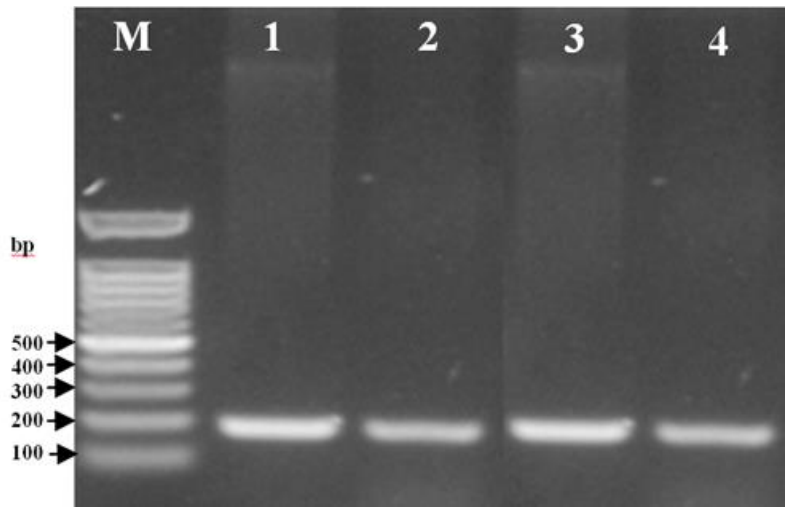

Figure 3: PCR amplification of oprl gene (202 bp), separated by electrophoresis on a $1 \%$ agarose gel, stained with red dye at 80 volts $/ \mathrm{cm}$ for 1 hour. Photographed under UV light. 1000 bp DNA marker (M); (1) $P$. aeruginosa CL1; (2) $P$. aeruginosa CL2; (3) $P$. aeruginosa CL3; (4) $P$. aeruginosa CL4.

Table 2: Comparison of biofilm biomass (OD550) by $P$. aeruginosa CL1, CL2, CL3 and CL4.

\begin{tabular}{|c|c|c|c|c|c|}
\hline $\begin{array}{l}P . \\
\text { aeruginosa } \\
\text { strain }\end{array}$ & CL1 & CL2 & CL3 & CL4 & $\begin{array}{l}\text { Negative } \\
\text { control }\end{array}$ \\
\hline $\begin{array}{l}\text { Absorbance } \\
\left(O_{550)}\right.\end{array}$ & $\begin{array}{c}1.06 \\
\pm \\
0.32\end{array}$ & $\begin{array}{c}1.93 \\
\pm \\
0.27\end{array}$ & $\begin{array}{c}0.35 \\
\pm \\
0.05\end{array}$ & $\begin{array}{c}0.25 \\
\pm \\
0.14\end{array}$ & $0.13 \pm 0.08$ \\
\hline
\end{tabular}

\section{Phage characteristics}

We screened water samples for phages infecting $P$. aeruginosa by using $P$. aeruginosa UKMCC 1011 strain as the host strain using double layer agar method. Six $P$. aeruginosa specific phages were isolated and labelled PAtk1, PAtk2, PAtk3, PAtk4, PAtk5 and PAtk6. PAtk2 formed clear plaques after $24 \mathrm{~h}$ of incubation at $37^{\circ} \mathrm{C}$, whilst the rest produced plaques with a smaller clear centre surrounded by halos.

The isolated phages were then observed through TEM (Philips CM12 TEM) with their morphological characteristics summarised in Table 3 . Based on the micrographs, PAtk1-5 was classified into the family Myoviridae due to the presence of contractile sheaths, meanwhile PAtk6 was classified into the family Siphoviridae due to the presence of long tails without contractile sheaths. Phages that were classified under Myoviridae have icosahedral heads and contractile sheaths. Basal plates for PAtk2, PAtk3, PAtk4 and PAtk5 were observed with diameters ranging from $49 \mathrm{~nm}$ to 77 $\mathrm{nm}$. PAtk3 had the longest size in length among the isolated Myoviridaes with total length of $300.63 \mathrm{~nm}$, whilst PAtk6 (family Siphoviridae) possessed long flexible tails with no contractile sheath and basal plate. Collar structure characteristics were observed in PAtk1, PAtk2, PAtk3 and PAtk4. All six phages were further tested against $P$. aeruginosa CL1, CL2, CL3 and CL4.

\section{Bacteriophage host spectrum}

Table 4 shows a summary of the host spectrum for the isolated phages. Amongst all six phages, PAtk4 and PAtk 6 showed the broadest lytic spectrum by showing lytic activity against all clinical $P$. aeruginosa strains. However, PAtk1 and PAtk2, alongside PAtk5 were unable to show lytic activity against $P$. aeruginosa CL4 and CL1, respectively. PAtk3 had the narrowest host range against the $P$. aeruginosa strains by exhibiting lytic activity on CL2 and CL3 only. Phage lytic ability was also screened against E. coli UKMCC 1006 and S. Typhi UKMCC 1013 but no lytic activity was observed, indicating the specificity of these phages against $P$. aeruginosa.

\section{DISCUSSION}

The increasing reports on worldwide multidrug resistance have raised concerns that led to the development of alternative therapeutical methods including phage therapy. $P$. aeruginosa is a major opportunistic pathogen that causes nosocomial and respiratory infections including those in cystic fibrosis (CF) patients (Pier, 1998). Thus, in this study, we isolated bacteriophages possessing lytic activity against clinical $P$. aeruginosa strains and investigated genotypic (exoS, toxA and oprl) and phenotypic (biofilm) virulence properties of the bacterial strains. The presence of tox $A$ has been found to correlate with antimicrobial resistance in Pseudomonas sp. (Amirmozafari et al., 2016), whilst exoS is highly important for invasion and colonisation (Bradbury et al., 2010) and oprl is associated with an outer membrane protein tethering to peptidoglycan in $P$. aeruginosa species (Wessel et al., 2013). 
Malays. J. Microbiol. Vol 14(6) Special Issue 2018, pp. 563-569 DOI: http://dx.doi.org/10.21161/mjm.1461815

Table 3: Morphological properties and family classification of isolated phages.

\begin{tabular}{lcccccc}
\hline \multirow{2}{*}{ Phage } & \multicolumn{5}{c}{ Particle size $(\mathrm{nm})$} & \multirow{2}{*}{ Family } \\
\cline { 2 - 6 } & Head & Tail length & Tail width & Base plate & Total length & Myoviridae \\
\hline PAtk1 & 105.44 & 164.68 & 31.83 & Absent & 270.12 & Myoviridae \\
PAtk2 & 104.64 & 128.09 & 25.03 & 51.87 & 232.73 & Myoviridae \\
PAtk3 & 113.64 & 186.99 & 26.40 & 77.51 & 300.63 & Myoviridae \\
PAtk4 & 106.64 & 126.58 & 25.06 & 58.66 & 233.22 & Myoviridae \\
PAtk5 & 108.72 & 137.38 & 24.60 & 49.20 & 246.1 & Siphoviridae \\
PAtk6 & 98.00 & 179.62 & 33.67 & Absent & 277.62 & \\
\hline
\end{tabular}

Table 4: Phage host spectrum on P. aeruginosa, E. coli and S. Typhi.

\begin{tabular}{|c|c|c|c|c|c|c|c|}
\hline \multirow{2}{*}{ Bacteria } & \multirow{2}{*}{ Type } & \multicolumn{6}{|c|}{ Phage } \\
\hline & & PAtk1 & PAtk2 & PAtk3 & PAtk4 & PAtk5 & PAtk6 \\
\hline \multirow[t]{4}{*}{ Pseudomonas aeruginosa } & CL1 & + & + & - & + & - & + \\
\hline & CL2 & + & + & + & + & + & + \\
\hline & CL3 & + & + & + & + & + & + \\
\hline & CL4 & - & - & - & + & + & + \\
\hline Escherichia coli & UKMCC 1006 & - & - & - & - & - & - \\
\hline Salmonella Typhi & UKMCC 1013 & - & - & - & - & - & - \\
\hline
\end{tabular}

+ , Iysis; - , no lysis

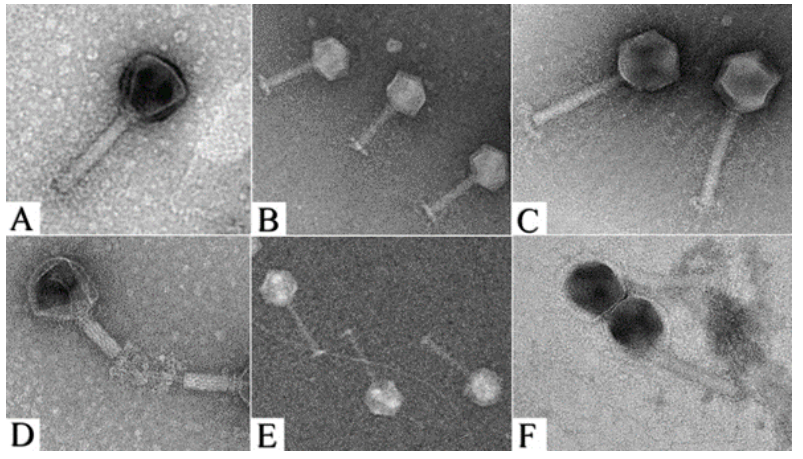

Figure 4: Electron micrographs demonstrating myoviral and siphoviral morphotypes for the isolated $P$. aeruginosa phages. The bar represents $200 \mathrm{~nm}$ length. A: PAtk1; B: PAtk2; C: PAtk3; D: PAtk4; E: PAtk5 and F: PAtk6.

Our investigation on clinical $P$. aeruginosa isolates showed that exoS, toxA and oprl were detected in all strains except for exoS in CL2. When comparing the biofilm production of these bacteria, CL2 produced the highest amount of biofilm biomass as shown from the absorbance readings. A study led by Azimi et al. (2016) discovered that exoS was only found in $2.5 \%$ of biofilmproducing $P$. aeruginosa isolates, implying that the gene is more prevalent in non-biofilm producing strains. Given that the exoenzyme $S$ regulon is activated by eukaryotic cell contact, $P$. aeruginosa biofilms lacking exoS should not be producing much of type III effectors encoded by the gene (Azimi et al., 2016)

Biofilm production is regarded as committed action and also an act of self-defence by bacterial communities that usually hinders the effectiveness of treatment towards bacteria (Drenkard, 2003; Weitere et al., 2005). In $P$. aeruginosa, the PA01 gene was observed to have an effect on its biofilm formation; however, it does not totally hinder formation but causes defective biofilm structure (Murphy et al., 2014). Therefore, instead of screening for biofilm-related genes, we investigated the status of these strains in forming biofilms. Hypothethically, strains with exoS, toxA and oprl with high efficiency of biofilm formation will be those that are highly virulent and resistant to antibiotics. Thus, in this study, we have chosen several clinical strains from a hospital, screened these strains for virulence genes, biofilm production and used these isolates as target for bacteriophage screening. This has enabled us to isolate bacteriophages that are highly virulent against antibiotic resistant Pseudomonas aeruginosa.

Bacteriophage morphology is one of the most important aspects of phage classification and therefore the characteristics of isolated phages were observed in this study. Six phages belong to the family Myoviridae and Siphoviridae under the order Caudovirales, where most $P$. aeruginosa bacteriophages are classified. Members of Caudovirales mostly consist of the typical head-and-tail morphology and contain double-stranded DNA (dsDNA) (Ackermann, 2011). Phages under Myoviridae have contractile tails while Siphoviridae consist of long, flexible ends (Aksyuk et al., 2009), that were observed in PAtk1 - PAtk5 and PAtk6, respectively. Nevertheless, PAtk1, PAtk2, PAtk3, PAtk4 and PAtk5 had slight differences in the size of their icosahedral head that could be due to errors in replicating viral DNA in these phages. Alterations in DNA due to mutations can result in 
Malays. J. Microbiol. Vol 14(6) Special Issue 2018, pp. 563-569 DOI: http://dx.doi.org/10.21161/mjm.1461815

errors during protein synthesis that may lead to a larger number of capsid proteins synthesised during gene expression. This can increase the phage head size even when they have similar genomes sequence and size. Further work on comparing genomes of phage with similar morphology is necessary to further address this possibility.

Amongst all isolated phages, PAtk4 and PAtk6 exhibited the broadest host range. The variety in lytic activity of these isolates against different $P$. aeruginosa strains could be attributed to the specificity of interaction between phage attachment structures and receptors on host cell surface (Yoon et al., 2007). The mechanism of host invasion by phage depends on a number of factors, including phage types, host cell structural material (e.g. glycoproteins and lipopolysaccharides), transport machinery and interactions between host cells through $F$ pili. Since PAtk4 and PAtk6 were the most virulent amongst the phages isolated, they should be further examined to determine their properties as potential antimicrobial agents against virulent strains of $P$. aeruginosa. Molecules that can be recognised by phages are essential components of the host cell surface (Sulakvelidze and Morris, 2001). Further characterisation is necessary to understand the intraspecific relationships among different types of $P$. aeruginosa and its phages. Some changes and alteration of host cell wall and membrane may affect the interaction between phage capsids and host membrane proteins, thus resulting in various degrees of successful infections. Lytic function of phage is the most crucial factor in phage therapy that advocates towards killing of pathogen (Woznica et al., 2015; Cisek et al., 2017). Lysogenic phages are unsuitable as it increases the resistance capability of target bacterium against antibiotics due to genetic material transfer (Balcazar, 2014).

\section{CONCLUSION}

In this study, we report the isolation of six bacteriophages specific to $P$. aeruginosa possessing exoS, toxA and opr genes. Five of the phages came from the family Myoviridae whilst one is from Siphoviridae. Phages PAtk4 and PAtk6 were the most virulent amongst all phages isolated, warranting further studies to determine and characterise their properties as potential antimicrobial agents against virulent strains of $P$. aeruginosa in vitro and in vivo.

\section{ACKNOWLEDGEMENTS}

The authors thank the Queen Elizabeth Hospital, Kota Kinabalu for providing clinical $P$. aeruginosa strains. We also want to thank the Department of Electron Microscopy, Universiti Kebangsaan Malaysia for providing the electron microscope for phage morphology observation.

\section{REFERENCES}

Ackermann, H. W. (2011). Phage or phages. Bacteriophage 1(1), 52-53.

Aksyuk, A. A., Leiman, P. G., Kurochkina, L. P., Shneider, M. M., Kostyuchenko, V. A., Mesyanzhinov, V. V. and Rossmann, M.G. (2009). The tail sheath structure of bacteriophage T4: A molecular machine for infecting bacteria. The EMBO Journal 28(7), 821-829.

Al-Kaaby, W. A. J. (2015). Molecular detection of virulence factors genes in Pseudomonas aeruginosa isolated from different infections cases in Al-Diwaniya Hospital. Al-Qadisiyha Journal for Science 2(20), 5358.

Amirmozafari, N., Fallah Mehrabadi, J. and Habibi, A. (2016). Association of the exotoxin A and exoenzyme $S$ with antimicrobial resistance. Archives of Iranian Medicine 19(5), 353-358.

Azimi, S., Kafil, H. S., Baghi, H. B., Shokrian, S., Najaf, K., Asgharzadeh, M. and Aghazadeh, M. (2016). Presence of exoY, exoS, exoU and exoT genes, antibiotic resistance and biofilm production among Pseudomonas aeruginosa isolates in Northwest Iran. GMS Hygiene and Infection Control 11, 1-6.

Balcazar, J. L. (2014). Bacteriophages as vehicles for antibiotic resistance genes in the environment. PLOS Pathogens 10(7), 1-4.

Bendiak, G. N. and Ratjen F. (2009). "The approach to Pseudomonas aeruginosa in cystic fibrosis." In: Seminars in Respiratory and Critical Care Medicine 30(05), 587-595.

Bradbury, R. S., Roddam, L. F., Merritt, A., Reid, D. W. and Champion, A. C. (2010). Virulence gene distribution in clinical, nosocomial and environmental isolates of Pseudomonas aeruginosa. Journal of Medical Microbiology 59(8), 881-890.

Breidenstein, E. B., de la Fuente-Núñez, C. and Hancock, R. E. (2011). Pseudomonas aeruginosa: All roads lead to resistance. Trends in Microbiology 19(8), 419-426.

Cisek, A. A., Dąbrowska, I., Gregorczyk, K. P. and Wyżewski, Z. (2017). Phage therapy in bacterial infections treatment: One hundred years after the discovery of bacteriophages. Current Microbiology 74(2), 277-283.

Drenkard, E. (2003). Antimicrobial resistance of Pseudomonas aeruginosa biofilms. Microbes and Infection 5(13), 1213-1219.

Fazeli, N. and Momtaz, H. (2014). Virulence gene profiles of multidrug-resistant Pseudomonas aeruginosa isolated from Iranian hospital infections. Iranian Red Crescent Medical Journal 16(10), 1-10.

Galle, M., Carpentier, I. and Beyaert, R. (2012). Structure and function of the Type III secretion system of Pseudomonas aeruginosa. Current Protein and Peptide Science 13(8), 831-842.

Holguín, A. V., Rangel, G., Clavijo, V., Prada, C., Mantilla, M., Gomez, ... and Vives, M. J. (2015). Phage ФPan70, a putative temperate phage, controls 
Malays. J. Microbiol. Vol 14(6) Special Issue 2018, pp. 563-569 DOI: http://dx.doi.org/10.21161/mjm.1461815

Pseudomonas aeruginosa in planktonic, biofilm and burn mouse model assays. Viruses 7(8), 4602-4623.

Holmes, D. S. and Quigley, M. (1981). A rapid boiling method for the preparation of bacterial plasmids. Analytical Biochemistry 114(1), 193-197.

Iglewski, B. H. and Kabat. (1975). NAD-dependent inhibition of protein synthesis by Pseudomonas aeruginosa. Proceedings of the National Academy of Sciences of the United States of America 72, 22842288.

Khan, A. A. and Cerniglia, C. E. (1994). Detection of Pseudomonas aeruginosa from clinical and environmental samples by amplification of the exotoxin A gene using PCR. Applied and Environmental Microbiology 60(10), 3739-3745.

Liu, P. V. (1974). Extracellular toxins of Pseudomonas aeruginosa. Journal of Infectious Diseases 130, 9499.

Murphy, K., Park, A. J., Hao, Y., Brewer, D., Lam, J. S. and Khursigaraa, C. M. (2014). Influence of $O$ polysaccharides on biofilm development and outer membrane vesicle biogenesis in Pseudomonas aeruginosa PAO1. Journal of Bacteriology 196(7), 1306-1317.

Nordmann, P., Naas, T., Fortineau, N. and Poirel, L. (2007). Superbugs in the coming new decade; multidrug resistance and prospects for treatment of Staphylococcus aureus, Enterococcus spp. and Pseudomonas aeruginosa in 2010. Current Opinion in Microbiology 10(5), 436-440.

O'Neill, J. (2014). Antimicrobial resistance: Tackling a crisis for the health and wealth of nations. Review on Antimicrobial Resistance. UK. pp. 1-20.

Pier, G. B. (1998). Pseudomonas aeruginosa: A key problem in cystic fibrosis. ASM News 64, 339-347.

Pollack, M. (1984). The virulence of Pseudomonas aeruginosa. Reviews of Infectious Diseases 6(3), 617626.

Sulakvelidze, A. and Morris, J. G. (2001). Bacteriophages as therapeutic agents. Annals of Medicine 38(3), 507-509.

Swanstrom, M. and Adams, M. H. (1951). Agar layer method for production of high titer phage stocks. Proceedings of the Society for Experimental Biology and Medicine 78(2), 372-375.

Ullah, W, Qasim, M., Rahman, H., Jie, Y. and Muhammad, N. (2017). Beta-lactamase-producing Pseudomonas aeruginosa: Phenotypic characteristics and molecular identification of virulence genes. Journal of the Chinese Medical Association 80(3), 173-177.

Viertel, T. M., Ritter, K. and Horz, H. P. (2014). Viruses versus bacteria-novel approaches to phage therapy as a tool against multidrug-resistant pathogens. The Journal of Antimicrobial Chemotherapy 69, 23262336.

Weitere, M., Bergfeld, T., Rice, S. A., Matz, G. and Kjelleberg, S. (2005). Grazing resistance of Pseudomonas aeruginosa biofilms depends on type of protective mechanism, developmental stage and protozoan feeding mode. Environmental Microbiology 7(10), 1593-1601.

Wessel, A. K., Liew, J., Kwon, T., Marcotte, E. M. and Whiteley, M. (2013). Role of Pseudomonas aeruginosa peptidoglycan-associated outer membrane proteins in vesicle formation. Journal of Bacteriology 195(2), 213-219.

Woznica, W. M., Bigos, J. and Lobocka, M. B. (2015). Lysis of bacterial cells in the process of bacteriophage release - canonical and newly discovered mechanisms. Postepy Higieny I Medycyny Doswiadczalnej (Online) 69(0), 114-126.

Yoon, S. S., Barrangou-Poueys, R., Breidt, F. and Fleming, H. P. (2007). Detection and characterization of a lytic Pediococcus bacteriophage from the fermenting cucumber brine. Journal of Microbiology and Biotechnology 17(2), 262-270.

Young, L. S. (1984). The clinical challenge of infections due to Pseudomonas aeruginosa. Reviews of Infectious Diseases 6(3), S603-S607. 